\title{
iNKT cells in chronic HBV: a balancing act
}

\author{
Maike Hofmann ${ }^{1} \cdot$ Robert Thimme $^{1}$
}

Received: 7 April 2016/Accepted: 8 May 2016/Published online: 24 May 2016

(C) Asian Pacific Association for the Study of the Liver 2016

Hepatitis B virus (HBV) is a major cause of chronic liver disease. Worldwide, more than 350 million people are chronically infected with HBV and therefore at risk of dying prematurely from liver failure or liver cancer. The pathogenesis of HBV-induced hepatitis is determined by the immune system. Thus, the natural clinical course of chronic HBV infection, which is defined by the persistent presence of HBsAg, is strictly linked to the immune response and can be subdivided in different phases [1]: first, the immunotolerant phase that is characterized by extremely high HBV-DNA, low transaminase levels and lack of anti-HBeAg in the sera of patients; second, the immunoactive phase with intermitting high HBV-DNA and transaminase levels; and third, a low-replicative phase, also called the inactive carrier phase, which is marked by low HBV-DNA, normal transaminases and the presence of anti$\mathrm{HBeAg}$. Although viral replication can be efficiently suppressed by current antiviral treatment strategies, chronic HBV infection remains largely incurable, as the virus can persist in the liver cell as a covalently closed circular DNA (cccDNA). Immunotherapy is considered a promising approach for HBV cure since HBV-specific immunity has been shown to determine the outcome and course of acute and chronic HBV infection. A prerequisite for successful immunotherapeutic approaches, however, is a detailed understanding of the role of the immune system in the different phases of chronic HBV infection.

Robert Thimme

robert.thimme@uniklinik-freiburg.de

1 Department of Medicine II, Clinic for Gastroenterology, Hepatology, Endocrinology and Infectious Diseases, University Hospital Freiburg, Freiburg, Germany
Intense research addressing immune responses in HBV infection has revealed that conventional $\mathrm{T}$ cells are the major effector cells in the elimination of $\operatorname{HBV}[2,3]$. In agreement with this, conventional $\mathrm{T}$ cell function is impaired in chronic HBV infection [4]. Effective $\mathrm{T}$ cell function depends on a proper induction of innate immune responses and an orchestrated cross-talk between the innate and adaptive immune system. Indeed, in the chimpanzee model, it has been observed that HBV infection does not strongly induce interferon stimulated genes, indicating a lack of early viral sensing by the immune system [5]. However, not much is known about the cross-talk of the innate and adaptive immune system in humans during HBV infection. Innate immune cells which bridge the gap between innate and adaptive immunity include natural killer T (NKT) cells. NKT cells are unconventional T cells that are subgrouped into type I, also known as invariant NKT (iNKT) cells, and type II, non-invariant NKT cells. Human iNKT cells are characterized by the expression of an invariant V $\alpha 24-J \alpha 18 / \mathrm{V} \beta 11 \mathrm{~T}$ cell receptor $[6,7]$. Upon antigenic stimulation by CD1d-presented lipid antigens (e.g. $\alpha$-GalCer), iNKT cells produce Th1, Th2 and Th17 cytokines and inflammatory mediators induce perforin expression rendering iNKT cells cytotoxic $[8,9]$.

In this issue, $\mathrm{Li} \mathrm{M}$. et al. compared frequencies and functional profiles of circulating iNKT cells derived from patients chronically infected with HBV, which has been categorized into immunotolerance $(n=16)$, inactive carrier $(n=25)$ and immunoactive phases $(n=19)$ [10]. In the immunotolerance phase of chronic HBV infection, a reduced frequency of iNKT among $\mathrm{CD} 3+\mathrm{T}$ cells was found compared to healthy controls. In contrast, chronically HBV-infected patients in the immunoactive and inactive carrier phases displayed increased levels of iNKT cells among $\mathrm{CD} 3+\mathrm{T}$ cells compared to healthy donors. 
These results extend previous data by Fisicaro et al., who reported high frequencies of activated iNKT cells early during HBV infection [11].

Interestingly, $\mathrm{Li} \mathrm{M}$. et al. also observed functional differences of iNKT cells derived from the different phases of HBV infection [10]. Indeed, while inactive carriers and healthy controls showed comparable levels of the Th1 cytokine IFN $\gamma$ and the Th2 cytokine IL-4, chronically HBV-infected patients in the immunotolerant and immunoactive phases displayed lower IFN $\gamma$ levels. However, it remains an open issue whether the impaired IFN $\gamma$ production of iNKT cells in immunotolerant and immunoactive HBV patients is due to a common mechanism or rather reflects immaturity of the immune system in the immunotolerant phase and exhaustion-like mechanisms in immunoactive HBV patients. It is important to note that a negative correlation between serum HBV-DNA and IFN $\gamma$-producing iNKT cells could be observed irrespective of the clinical phase of chronic HBV infection. In contrast, IL-4 production by iNKT cells positively correlated with serum HBV-DNA of patients chronically infected with HBV. In line with this, an increased proportion of iNKT cells derived from immunotolerant and immunoactive HBV patients produced IL-4 compared to inactive carriers and healthy donors. These results suggest that iNKT cells from chronic HBV patients are not generally impaired in their function but rather possess an imbalanced functionality (Fig. 1). Whether this functional alteration results from a redistribution of iNKT cell subsets [12] or from distinct (re-)differentiation due to persisting antigenic stimulation or chronic exposure to inflammatory mediators remains an open question. Of note, it has been shown that iNKT cells can be activated both by IL-12 in a HBV mouse model and by ER-derived phospholipid antigens that are induced upon HBV infection of murine and human hepatocytes in vitro [13]. Additionally, Li M. et al. observed minor differences comparing patients chronically infected with HBV before and under entecavir treatment with a slight increase in IFN $\gamma$-producing and a decrease in IL-4producing iNKT cells [10]. These results possibly reflect plasticity of iNKT cells, either on a single cell or on a

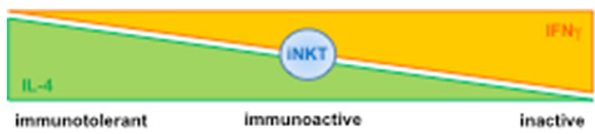

Fig. 1 Functional alterations of iNKT cells in chronic HBV infection. In healthy donors, a large proportion of circulating iNKT cells produces IFN $\gamma$. This proportion of IFN $\gamma$-producing iNKT cells is reduced in the blood of patients chronically infected with HBV in the immunotolerance and immunoactive phases, which are characterized by high viral loads. In inactive $\mathrm{HBsAg}$ carriers, however, the functional profile of circulating iNKT cells is similar to healthy donors with respect to IFN $\gamma$ and IL4 production population level, in chronic HBV infection that is influenced by viral load.

The interest in targeting iNKT cells in immunotherapy in the context of liver disease is based on the fact that iNKT cells represent $10-15 \%$ of liver lymphocytes in humans $[14,15]$. Furthermore, iNKT cells have well-described modulatory effects on CD8 $+\mathrm{T}$ cells, the main effector cells in the elimination of HBV [16]. These modulatory effects comprise indirect measures such as licensing of dendritic cells that then in turn cross-present antigen to CD8+ T cells as well as having direct influences on $\mathrm{CD} 8+\mathrm{T}$ cells via cytokines. Interestingly, in HBV mouse models, for example, it has been described that activation of iNKT cells can enhance $\mathrm{T}$ cell-mediated immunity and can lead to HBV elimination $[17,18]$. Yet, in a clinical trial, intravenous administration of $\alpha$-GalCer, a well-described prototypic antigen recognized by iNKT cells, failed to induce a protective effect in chronic HBV patients [19]. According to these inconsistent results, accumulating evidence suggests dual or even opposing roles of iNKT cells in liver pathology, like injury and regeneration or fibrosis and tumor development. This is, for example, illustrated by the protective role of iNKT cells in mouse models of acute liver injury, whereas in chronic conditions iNKT cells can promote liver injury [20]. The mechanisms responsible for this complex role of iNKT cells in liver pathologies are not completely understood but include the secretion of a variety of cytokines by iNKT that have different functions and can act synergistically depending on other factors, for example environmental factors. Environmental factors include mediators such as cytokines induced by surrounding non-resident cells and tissue-specific signals by resident and stoma cells. These factors also influence the cytokine pattern secreted by iNKT cells and thus establish a complex feedback loop. This demonstrates that iNKT-cell function and the consequent effects are versatile and context-dependent, and therefore modulation of iNKT cells, for example by specific antigenic stimuli and/or cytokine combinations, has to be exactly balanced in order to obtain beneficial effects in immunotherapy in liver diseases such as chronic HBV infection.

In sum, $\mathrm{Li} \mathrm{M}$. et al. demonstrate differences in the functional immune profile of iNKT cells during the different phases of chronic HBV infection [10], emphasizing the importance of taking dynamic aspects of the clinical course into account when analyzing the immune response in HBV with the aim of defining targets and establishing strategies for immunotherapy in HBV infection. Moreover, this study also suggests that the interface between the innate and adaptive arm of the immune system is probably an important rheostat in balancing immune responses in viral hepatitis. This is even further supported by the 
emerging findings about the role of other lymphoid cells in healthy and diseased livers that bridge the gap between innate and adaptive immunity, for example MAIT cells and innate lymphoid cells [21].

\section{Compliance with ethical standards}

Conflict of interest M. Hofmann and R. Thimme declare that they have no conflict of interest.

\section{References}

1. Rehermann B. Pathogenesis of chronic viral hepatitis: differential roles of T cells and NK cells. Nat Med 2013;19:859-868

2. Ferrari C. HBV and the immune response. Liver Int 2015;35 Suppl 1:121-128

3. Thimme R, Wieland S, Steiger C, et al. CD8(+) T cells mediate viral clearance and disease pathogenesis during acute hepatitis $\mathrm{B}$ virus infection. J Virol 2003;77:68-76

4. Boni C, Fisicaro P, Valdatta C, et al. Characterization of hepatitis B virus (HBV)-specific T-cell dysfunction in chronic HBV infection. J Virol 2007;81:4215-4225

5. Wieland SF, Spangenberg HC, Thimme R, et al. Expansion and contraction of the hepatitis B virus transcriptional template in infected chimpanzees. Proc Natl Acad Sci USA 2004;101:2129-2134

6. Dellabona P, Padovan E, Casorati G, et al. An invariant V alpha 24-J alpha $\mathrm{Q} / \mathrm{V}$ beta $11 \mathrm{~T}$ cell receptor is expressed in all individuals by clonally expanded CD4-8- T cells. J Exp Med 1994;180:1171-1176

7. Lantz O, Bendelac A. An invariant T cell receptor alpha chain is used by a unique subset of major histocompatibility complex class I-specific CD4+ and CD4-8- T cells in mice and humans. J Exp Med 1994;180:1097-1106

8. Gumperz JE, Miyake S, Yamamura T, et al. Functionally distinct subsets of CD1d-restricted natural killer $\mathrm{T}$ cells revealed by CD1d tetramer staining. J Exp Med 2002;195:625-36
9. Michel ML, Keller AC, Paget C, et al. Identification of an IL-17producing NK1.1(neg) iNKT cell population involved in airway neutrophilia. J Exp Med 2007;204:995-1001

10. Li M, et al. The dynamic changes of circulating invariant natural killer T cells during chronic hepatitis B virus infection. Hepatol Int. 2016. doi:10.1007/s12072-015-9650-0.

11. Fisicaro P, Valdatta C, Boni C, et al. Early kinetics of innate and adaptive immune responses during hepatitis B virus infection. Gut 2009;58:974-982

12. Gapin L. Development of invariant natural killer T cells. Curr Opin Immunol 2016;39:68-74

13. Zeissig S, Murata K, Sweet L, et al. Hepatitis B virus-induced lipid alterations contribute to natural killer $\mathrm{T}$ cell-dependent protective immunity. Nat Med 2012;18:1060-1068

14. Duwaerts CC, Gregory SH. Targeting the diverse immunological functions expressed by hepatic NKT cells. Expert Opin Ther Targets 2011;15:973-988

15. Gao B, Radaeva S, Park O. Liver natural killer and natural killer $\mathrm{T}$ cells: immunobiology and emerging roles in liver diseases. J Leukoc Biol 2009;86:513-528

16. Gottschalk C, Mettke E, Kurts C. The role of invariant natural killer $\mathrm{T}$ cells in dendritic cell licensing, cross-priming, and memory CD8(+) T cell generation. Front Immunol 2015;6:379

17. Ito H, Ando K, Ishikawa T, et al. Role of Valpha14+ NKT cells in the development of Hepatitis B virus-specific CTL: activation of Valpha14+ NKT cells promotes the breakage of CTL tolerance. Int Immunol 2008;20:869-879

18. Kakimi K, Guidotti LG, Koezuka Y, et al. Natural killer T cell activation inhibits hepatitis B virus replication in vivo. J Exp Med 2000;192:921-930

19. Cerundolo V, Silk JD, Masri SH, et al. Harnessing invariant NKT cells in vaccination strategies. Nat Rev Immunol 2009;9:28-38

20. Wang H, Feng D, Park O, et al. Invariant NKT cell activation induces neutrophil accumulation and hepatitis: opposite regulation by IL-4 and IFN-gamma. Hepatology 2013;58:1474-1485

21. Heymann F, Tacke F. Immunology in the liver-from homeostasis to disease. Nat Rev Gastroenterol Hepatol 2016;13:88-110 